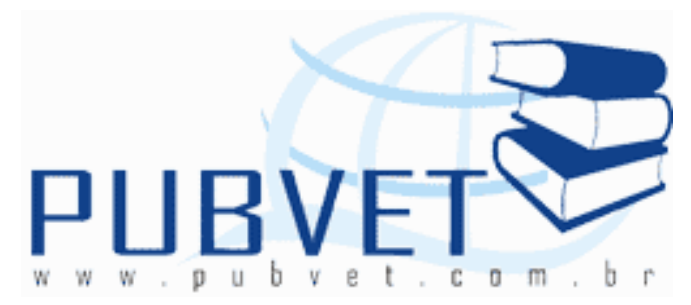

PUBVET, Publicações em Medicina Veterinária e Zootecnia.

\title{
Avaliação dos efeitos de diferentes doses de etomidatos na contenção farmacológica de cágado-de-barbicha Phrynops geoffroanus SCHWEIGGER, 1812 (Testudines, Chelidae)
}

André Luiz Quagliatto Santos ${ }^{1}$, Lívia monteiro Magalhães ${ }^{2}$, Flávio Machado de Morais ${ }^{1}$, Rogério Rodrigues de Souza ${ }^{2}$, Lorena Tannús Menezes ${ }^{2}$, Caio Henrique Ferreira $^{2}$, Sérgio Rodrigo Pereira de Oliveira², Liliane Rangel Nascimento², Mariana Batista Andrade ${ }^{2}$.

Laboratório de Ensino e Pesquisas em Animais Silvestres - LAPAS, FAMEV/UFU, e-mail: quagliatto@famev.ufu.br 1. Docente. 2. Mestrandos 2. Médica Veterinária Autônoma.

\section{Resumo}

O objetivo deste trabalho foi avaliar os efeitos anestésicos de diferentes protocolos de etomidato em Phyrynops geoffroanus. Foram utilizadas 30 Phrynops geoffraonus (cágado-de-barbicha), provenientes do rio Uberabinha, município de Uberlândia, MG, clinicamente saudáveis, sendo 17 machos e 13 femeas, e peso variando de 0,400 a 3,550 quilogramas. A pesquisa foi realizada no mês de novembro de 2006, em que as condições climáticas eram de temperatura amena e clima úmido. Os cágados foram divididos em grupos de dez e anestesiados com diferentes protocolos de etomidatos: o grupo 1 (G1) recebeu 1,0mg/kg IV; o grupo 2 (G2) recebeu $1,5 \mathrm{mg} / \mathrm{kg}$ IV o grupo 3 (G3) recebeu $3,0 \mathrm{mg} / \mathrm{kg}$ IV. A administração do anestésico foi feita por via 
direta no seio vertebral cervical. Observaram-se os parâmetros anestésicos: locomoção, relaxamento muscular, resposta aos estímulos nos membros torácicos e pelvinos, manipulação e batimentos cardíacos, nos tempos $0,5,10$, 20, 30, 40, 45, 60, 90, 120, 150 e 180 minutos após as injeções. Conclui-se que o etomidato, na dose de $3,0 \mathrm{mg} / \mathrm{Kg} \mathrm{IV}$, embora seguro não foi eficiente para anestesia cirúrgica de Phrynops geoffroanus, sendo indicado para ocasiões que necessitam de uma satisfatória contenção farmacológica, com temperatura ótima; e que nas doses de $1,0 \mathrm{mg} / \mathrm{Kg}$ IV e $1,5 \mathrm{mg} / \mathrm{Kg}$ IV é indicado em situações que exijam sedação satisfatória em Phrynops geoffroanus, como procedimentos clínicos e coleta de amostras biológicas, em temperatura ótima.

Palavras-chave: Etomidato, phrynops geoffroanus, Cágado-de-barbicha, Répteis.

\section{Evaluation of the effects of different doses of etomidate pharmacology contention on Phrynops geoffroanus SCHWEIGGER, 1812 (Testudines, Chelidae)}

\section{Summary}

The objective of this study was to evaluate the effects of different anesthetic protocols in Phyrynops geoffroanus etomidate. We used 30 Phrynops geoffraonus (turtle-de-beard), Uberabinha from the river, Uberlândia, MG, clinically healthy, 17 males and 13 females, and weight ranging from 0.400 to 3.550 kilograms. The survey was conducted in November 2006, where climatic conditions were warm and humid climate. The tortoises were divided into groups of ten anesthetized with etomidate different protocols: group 1 (G1) received $1.0 \mathrm{mg} / \mathrm{kg}$ IV, Group 2 (G2) received $1.5 \mathrm{mg} / \mathrm{kg}$ IV group 3 (G3) received $3.0 \mathrm{mg} / \mathrm{kg}$ IV. The administration of the anesthetic was made by direct cervical spine within. Parameters were observed anesthetic locomotion, muscle relaxation, response to stimuli in the thoracic limbs and pelvinos, handling and heartbeat, at 0, 5, 10, 20, 30,40,45, 60, 90, 120, 150 and 180 
minutes after the injections. It is concluded that etomidate at a dose of $3.0 \mathrm{mg}$ / kg IV, although insurance was not effective for surgical anesthesia Phrynops geoffroanus It is suitable for occasions that require a satisfactory pharmacological restraint, with optimum temperature, and that doses of 1.0 $\mathrm{mg} / \mathrm{kg}$ IV and $1.5 \mathrm{mg} / \mathrm{kg}$ IV is indicated in situations that require sedation satisfactory Phrynops geoffroanus as clinical procedures and collection of biological samples at optimum temperature.

Keywords: Etomidate, phrynops geoffroanus, Tortoise-of-goatee Reptiles.

\section{INTRODUÇÃO}

A ordem Testudines é constituída por tartarugas, cágados e jabutis. A principal característica dessa ordem é o casco que reveste o corpo do animal, formado por carapaça (dorsal) e plastrão (ventral). Esses animais possuem diferentes habitats: espécies exclusivamente terrestres como os jabutis (Geochelonia sp); espécies exclusivamente marinhas como as tartarugas (Caretta caretta), e ainda, espécies que vivem em ambientes terrestre e de água doce como a Aperema (Rhynoclemmys punctlariaia) (GARCIA NAVARRO; PACHALY, 1994).

Os cágados possuem carapaças baixas que oferecem pequena resistência ao deslocamento na água, e patas anteriores modificadas em remos auxiliando sua locomoção (POUCH; HEISER; MCFARLAND, 1999).

A Phrynops geoffroanus é popularmente conhecida como cágado de barbicha e possui carapaça achatada e larga com coloração preta ou cinza esverdeada. O plastrão possui um entalhe anal e tem uma coloração vermelha rosada com manchas pretas irregulares. A cabeça é larga e lisa, com coloração preta, linhas brancas e irregulares. A parte inferior é branca com linhas e pontos pretos. A característica mais proeminente dessa espécie é uma linha preta e larga que se estende longitudinalmente pelo olho, também possui um par de "barbelas" no queixo com a extremidade preta. É uma espécie pequena encontrada desde a Colômbia, Venezuela, Guiana, extremo sul do Paraguai até 
sudeste, centro-oeste e nordeste do Brasil, sendo frequentemente nos rios, lagos e lagos com correnteza lenta (ERNESTE; BARBOUR, 1989).

A utilização de anestésicos faz-se necessária para a realização de exames físicos, procedimentos clínicos e cirúrgicos na medicina de répteis. No entanto, muito do que se conhece em anestesia destes animais, é baseado em impressões da clinica e casos apresentados por veterinários com experiência em répteis. Pouco é conhecido sobre os efeitos dos anestésicos nestes animais, em parte, por causa dos ramos estudos e investigações (HINSCH; GANDAL, 1969; KANUI; HOLE, 1992).

$\mathrm{Na}$ década de 70 e inicio da década de 80 esses animais eram submetidos à imobilização e anestesia através da hipotermia e inalação de éter, sendo que atualmente considere-se que essas técnicas são ineficazes, de alto risco e para alguns até desumanas (BENNETT, 1996). Na medida em que se aprofundam os conhecimentos anatômicos, fisiológicos e de anestesia dos répteis, é possível desenvolver protocolos eficazes e seguros.

Bennett (1991) e Boyer (1992), ao estudarem anestesia em quelônios, observaram que muitas técnicas foram testadas, mas a maioria apresentava limitações e efeitos colaterais, demonstrando que conhecimentos de sedação e anestesia são ilimitados nesses animais.

Nos últimos anos, alguns trabalhos vêm sendo publicados com a utilização de etomidato na anestesia de répteis. Avila Junior (2005) anestesiou 60 Podocnemis expansa (tartaruga da Amazônia) utilizando diferentes protocolos, dentre eles: etomidato, etomidato associado com butorfanol e etomidato com fentanila.

Este trabalho teve como objetivo avaliar os efeitos anestésicos de diferentes protocolos de etomidato em Phrynops geoffroanus, visando determinar qual o mais seguro, eficiente e adequado para cada situação clínica e cirúrgica que envolva a espécie em questão. 


\section{MATERIAL E MÉTODO}

Foram utilizadas 30 Phrynops geoffroanus (cágado-de-barbicha), proveniente do rio Uberabinha, $\left(18^{\circ} \mathrm{C} 55^{\prime} 42,1^{\prime \prime} \mathrm{S}\right.$ e $48 \mathrm{C}$ e $\left.17^{\prime} 35,4^{\prime \prime} \mathrm{W}\right)$, município de Uberlândia, MG, sob licença No 032-2006 IBAMA-RAN e No 139/11 da comissão de ética na utilização de animais, clinicamente saudáveis, 17 machos e 13 fêmeas, e peso variando de 0,400 a 3,550 kg. O experimento foi conduzido no laboratório de pesquisa em animais silvestres (LAPAS) da Universidade federal de Uberlândia, Uberlândia MG.

A pesquisa foi realizada no mês de novembro de 2006, em que as condições climáticas eram de temperatura amena e clima úmido sendo que a temperatura oscilou entre 28 e 30 graus Celsius. A temperatura ambiental era aferida a cada hora, com termômetro de máxima e mínima ${ }^{1}$.

No Lapas, os Animais foram mantidos em uma piscina de fibra de vidro com água aquecida por aquecedores elétricos. Os espécimes foram pesados em balanças pesadoras simples ${ }^{2}$ e identificados no casco com numeração sequencial. Antes de realizar o experimento, foram aferidos os batimentos cardíacos de todos os repteis.

A administração do anestésico foi feita por via direta no seio vertebral cervical, mediante previa antissepsia e uso de seringas estéreis ${ }^{3}$ de $3 \mathrm{ml}$, acopladas e agulhas hipodérmicas ${ }^{4} 25 \times 0,7 \mathrm{~mm}$.

Os cágados foram divididos em grupos de dez e anestesiados com diferentes protocolos de etomidatos ${ }^{5}$ : o grupo 1 (G1) recebeu $1,0 \mathrm{mg} / \mathrm{kg} \mathrm{IV;} \mathrm{o}$ grupo 2 (G2) recebeu $1,5 \mathrm{mg} / \mathrm{kg}$ IV o grupo 3 (G3) recebeu $3,0 \mathrm{mg} / \mathrm{kg} \mathrm{IV.}$

Os parâmetros foram observados dos determinados tempos $5,10,20$, $30,45,60,90,120,150,180$ minutos; sendo que tempo zero foi considerado o momento da administração da droga. Os parâmetros avaliados foram os seguintes:

Locomoção: capacidade de se locomover novamente, dificuldade de locomoção e ausência de locomoção; 
Relaxamento muscular: capacidade de manter a cabeça elevada, facilidade de estender os membros do animal;

Manipulação: facilidade de manipular manualmente (flexão e extensão) a cabeça, os membros, a cauda e a boca do animal;

Resposta do estimulo dos membros torácicos e pelvinos: pinçamento das falanges com pinça hemostática Kelly reta de $16 \mathrm{~cm}$, na primeira trava;

Batimentos cardíacos: aferição dos batimentos cardíacos Doppler vascular $^{6}$ nos tempos $0,10,30,60,120$ e 180 minutos.

Um escore subjetivo de um para efeito mínimo, 2 para efeito médio e 3 para Máximo efeito foi utilizado para os três parâmetros. Para os testes de respostas aos estímulos dos membros, a resposta ao pinçamento foi considerado escore 0 e, ausência da resposta do pensamento foi considerado esocre 1.

Os escores eram anotados em fichas anestesiológicas individuais (quadro 1).

Os repteis foram considerados anestesiados quando o somatório do escore foi 11; Avila Junior (2005) e considerados recuperados quando suas atividades aproximavam as de antes da administração das drogas. O tempo entre a administração dos anestésicos e a recuperação dos animais foi observado, considerados como retorno.

A analise estatística foi feita levando-se em consideração quando tempo (minutos) os animais permaneceram em escore três, em escore dois e quanto tempo eles gastaram para retornar ao escore um aos parâmetros locomoção, relaxamento muscular e manipulação.

Para os parâmetros resposta ao estímulo dos membros torácicos e pelvinos, levou-se em conta quanto tempo os espécimes mantiveram-se em escore um, e quanto tempo demoraram a voltar para o escore zero.

Os resultados foram avaliados utilizando a analise de variância (BANZATTO; KRONKA, 1995) para verificar a ocorrência de diferenças significativas entre os tratamentos. Ocorrendo, utilizou-se 0 teste de 
comparação múltiplas de Tukey a 5\% de probabilidade (BANZATTO; KRONKA, 1995).

Foram confeccionados fotografias para ilustração do teste de resposta aos estímulos aos membros (fotografia 1 e 2), do nível de relaxamento muscular (Fotografia 3 e 4), facilidade de manipulação (Fotografia 5), da aferição dos batimentos cardíaco (fotografia 6).

\section{RESULTADOS}

Todos os répteis estudados obtiveram escore três (grau máximo de efeito) para os parâmetros locomoção e relaxamento muscular, em até dois minutos após a administração do anestésico. O G3 foi o único grupo onde todos os animais entraram em anestesia, ou, seja obtiveram escore 11 . No G1 e G2 nenhum dos cágados entrou em anestesia. No entanto, todos os animais do G2 atingiram escore três também para o parâmetro manipulação, em até dois minutos após a administração do etomidato. Apenas quatro animais do G1 obtiveram escore três para manipulação.

Em relação á resposta ao estimulo do membro torácico, no $\mathrm{G} 2$ todos os animais alcançaram escore um, ou seja, ausência de resposta ao estímulo; enquanto que no $\mathrm{G} 1$ oito repteis alcançaram escore um para o mesmo parâmetro. Em ambos os grupos ( $\mathrm{G} 1$ e G2) todos os animais mantiveram-se em escore zero para resposta ao estimulo do membro pelvino, ou seja, respondiam ao estímulo quando pinçados.

Todos os répteis foram acompanhados por 48 horas depois da administração do anestésico e nenhum animal veio a óbito.

Verificou-se que o G1, o G2 e o G3 apresentaram em media 93, 132 e 196 minutos respectivamente de tempo de recuperação. No G3, um animal recuperou-se da anestesia depois de 24 horas após administração do etomidato, tempo muito superior aos apresentados pelos outros cágados do mesmo grupo. 

farmacológica de cágado-de-barbicha Phrynops geoffroanus SCHWEIGGER, 1812 (Testudines, Chelidae). PUBVET, Londrina, V. 6, N. 12, Ed. 199, Art. 1335, 2012.

Tabela 1 - Resumo da analise de variância para as variáveis TLoc3 (tempo em que os animais permaneceu em escore três para locomoção), TLoc2 (tempo em que o animal permaneceu em escore dois para locomoção) e TVLoc1 (tempo que o animal demorou a voltar ao escore um para locomoção).

\begin{tabular}{|l|c|c|c|c|}
\hline & & \multicolumn{3}{|c|}{ Quadrado Médio } \\
\hline F.V. & G.L. & TLoc 3 & TLoc 2 & TVLoc1 \\
\hline Trat. & 2 & $4729,65^{*}$ & $3514,22 *$ & $20873,62 *$ \\
\hline Res. & 26 & 621,92 & 818,26 & 860,38 \\
\hline CVE & & $29,53 \%$ & $28,78 \%$ & $11,63 \%$ \\
\hline
\end{tabular}

Para analise os dados foram transformados para $\sqrt{ } x+0,5$.

*Diferenças significativas ao nível de probabilidade de 0,05 .

Para o parâmetro colocação, pode ser observar que os tratamentos apresentaram diferenças estatísticas significativas entre si em relação ao tempo em que os animais permaneceram em escore três e dois e no tempo que os espécimes demoraram a retornar ao escore um para o parâmetro locomoção.

Tabela 2 - Estimativa de medias para as variáveis TLoc 3 (tempo em que os animais permaneceram em escore 3 para locomoção). TLoc 2 (tempo em que o animal permaneceu em escore dois para locomoção) e TVLoc1 (tempo que o animal demorou a voltar ao escore um para locomoção).

\begin{tabular}{|l|c|c|c|}
\hline & \multicolumn{3}{|c|}{ Médias } \\
\hline Trat. & TLoc3 & TLoc2 & TVLoc1 \\
\hline T1,0 mg & $17 \mathrm{a}$ & $34,5 \mathrm{a}$ & $93 \mathrm{a}$ \\
\hline T1,5mg & $37 \mathrm{a} \mathrm{b}$ & $43,5 \mathrm{~b}$ & $132 \mathrm{~b}$ \\
\hline T3,0mg & $61 \mathrm{~b}$ & $71,6 \mathrm{~b}$ & $186 \mathrm{c}$ \\
\hline
\end{tabular}

Para analise os dados foram transformados para $\sqrt{ } x+0,5$.

Medias seguida de letras iguais não diferem estatisticamente pelo teste tukey a $5 \%$ de probabilidade.

De acordo com a tabela 2, para as variáveis, TLoc 3 e TLoc 2, 0 tratamento utilizado no $\mathrm{G} 1(1,0 \mathrm{mg} / \mathrm{Kg})$ não difere estatisticamente do utilizado 
no $\mathrm{G} 2(1,5 \mathrm{mg} / \mathrm{Kg})$; o tratamento realizado no $\mathrm{G} 2$ não difere estatisticamente do utilizado no $\mathrm{G} 3(3,0 \mathrm{mg} / \mathrm{Kg})$ e o tratamento utilizado no $\mathrm{G} 1$ difere estatisticamente do utilizado no G3.

Para a variável TVLoc1, todos os tratamentos diferem-se estatisticamente entre si.

Tabela 3 - Resumo da analise de variância para as variáveis TRM 3 (tempo em que os animais permaneceram em escore três para relaxamento muscular), TRM 2 (tempo em que o animal permaneceu em escore dois para relaxamento muscular), TVRM1 (tempo que o animal demorou a voltar ao escore um para relaxamento muscular).

\begin{tabular}{|l|c|c|c|c|}
\hline & & \multicolumn{3}{|c|}{ Quadrado Médio } \\
\hline F.V. & G.L. & TRM3 & TRM2 & TVRM1 \\
\hline Trat. & 2 & $2112,93^{*}$ & $8641,85^{*}$ & $21638,23 *$ \\
\hline Res. & 26 & 488,46 & 646,25 & 826,63 \\
\hline CVE & & $30,36 \%$ & $25,13 \%$ & $11,85 \%$ \\
\hline
\end{tabular}

Para analise os dados foram transformados para $\sqrt{ } \mathrm{x}+0,5$.

*Diferenças significativas ao nível de probabilidade de 0,05 e ns não difere estatisticamente.

Houve diferenças significativas entre tratamento em relação ao tempo de permanência dos animais em escore três e dois e o tempo gasto para os repteis retornarem ao escore para o parâmetro relaxamento muscular.

De acordo com a tabela 4, para a variável TRM3 o G1 não difere estaticamente do G2 e este por sua vez, não difere estatisticamente do G3.

Para a variável TRM2, o G1 e G2 não diferem estatisticamente entre si. Já o G3 apresenta diferença estatística em relação aos demais.

Para a variável TVRM1, os três grupos apresentam diferenças estatísticas entre si. 

farmacológica de cágado-de-barbicha Phrynops geoffroanus SCHWEIGGER, 1812 (Testudines, Chelidae). PUBVET, Londrina, V. 6, N. 12, Ed. 199, Art. 1335, 2012.

Tabela 4 - Estimativa de medias para as variáveis TRM 3 (tempo em que os animais permaneceram em escore 3 para relaxamento muscular). TRM 2 (tempo em que o animal permaneceu em escore dois para relaxamento muscular), TVRM1 (tempo que o animal demorou a voltar ao escore um para relaxamento muscular).

\begin{tabular}{|l|c|c|c|}
\hline & \multicolumn{3}{|c|}{ Medias } \\
\hline Trat. & TRM3 & TRM2 & TVRM1 \\
\hline T1,0 mg & $17 \mathrm{a}$ & $32 \mathrm{a}$ & $91,5 \mathrm{a}$ \\
\hline T1,5mg & $34 \mathrm{a} \mathrm{b}$ & $44 \mathrm{~b}$ & $129 \mathrm{~b}$ \\
\hline T3,0mg & $46 \mathrm{~b}$ & $90 \mathrm{~b}$ & $186,6 \mathrm{c}$ \\
\hline
\end{tabular}

Para analise os dados foram transformados para $\sqrt{ } \mathrm{x}+0,5$.

Medias seguida de letras iguais não diferem estatisticamente pelo teste tukey a $5 \%$ de probabilidade.

Tabela 5 - Resumo da analise de variância para as variáveis TEMT 1 (tempo em que o animal permaneceu em escore um para resposta ao estimulo do membro torácico), TVEMTO (tempo em que o animal demorou a voltar ao escore zero para resposta ao estimulo do membro torácico).

\begin{tabular}{|l|c|c|c|}
\hline & \multicolumn{3}{|c|}{ Quadrado Médio } \\
\hline F.V. & G.L. & TEMT1 & TVEMTO \\
\hline Trat. & 2 & $4562,99 \mathrm{~ns}$ & $6772,02 \mathrm{~ns}$ \\
\hline Res. & 26 & 1746,94 & 2540,48 \\
\hline CVE & & $45,53 \%$ & $37,45 \%$ \\
\hline
\end{tabular}

Para analise os dados foram transformados para $\sqrt{ } x+0,5$.

*Diferenças significativas ao nível de probabilidade de o,05.

A tabela acima exibe que não há diferenças estatísticas entre os tratamentos, levando-se em conta as variáveis TEMT1 e TVEMTO. 

farmacológica de cágado-de-barbicha Phrynops geoffroanus SCHWEIGGER, 1812 (Testudines, Chelidae). PUBVET, Londrina, V. 6, N. 12, Ed. 199, Art. 1335, 2012.

Tabela 6 - Estimativas de médias para as variáveis TEMT1 (tempo que o animal permaneceu em escore um para resposta ao estimulo do membro torácico), TVEMTO (tempo que o animal demorou a voltar ao escore zero para resposta ao estimulo do membro torácico).

\begin{tabular}{|l|c|c|}
\hline & \multicolumn{2}{|c|}{ Médias } \\
\hline Trat. & TEMT1 & TVEMT0 \\
\hline T1,0 mg & $33,5 \mathrm{a}$ & $52,5 \mathrm{a}$ \\
\hline T1,5mg & $57,2 \mathrm{a}$ & $86,0 \mathrm{a}$ \\
\hline T3,0mg & $77,22 \mathrm{a}$ & $105 \mathrm{a}$ \\
\hline
\end{tabular}

Para analise os dados foram transformados para $\sqrt{ } \mathrm{x}+0,5$.

Medias seguida de letras iguais não diferem estatisticamente pelo teste tukey a $5 \%$ de probabilidade.

Conforme a tabela 6, o G1 o G2 e o G3 não diferem estatisticamente entre si em relação ao tempo de permanecia dos animais em escore um e o tempo gasto de volta dos mesmos ao escore zero para o parâmetro de resposta ao estimulo do membro torácico.

Tabela 7 - Resumo da analise de variância para as variáveis TEMP 1 (tempo em que os animais permaneceu em escore um para resposta ao estimulo do membro pelvino). TVEMPO (tempo que o animal demorou a voltar ao escore zero para resposta ao estimulo do membro pelvino).

\begin{tabular}{|l|c|c|c|}
\hline & \multicolumn{3}{|c|}{ Quadrado Médio } \\
\hline F.V. & G.L. & TEMP1 & TVEMPO \\
\hline Trat. & 2 & $1456,86^{*}$ & $4965,51^{*}$ \\
\hline Res. & 26 & 53,84 & 121,15 \\
\hline CVE & & $34,35 \%$ & $30,61 \%$ \\
\hline
\end{tabular}

Para analise os dados foram transformados para $\sqrt{ } \mathrm{x}+0,5$.

*Diferenças significativas ao nível de probabilidade de 0,05

Nota-se que os tratamentos apresentam diferenças significativas entre si em relação ao tempo de permanecia dos animais em escore um tempo que os repteis utilizaram para retornam ao escore zero para o parâmetro de resposta ao estímulo do membro pelvino. 
Tabela 8 Estimativas de medias para as variáveis TEMP1 (tempo em que o animal permaneceu em escore um para resposta ao estimulo do membro pelvino). TVEMPO (tempo que o animal demorou a voltar ao escore zero para resposta ao estímulo do membro pelvino).

\begin{tabular}{|l|c|c|}
\hline & \multicolumn{2}{|c|}{ Médias } \\
\hline Trat. & TEMP1 & TVEMPO \\
\hline T1,0 mg & $0,0 \mathrm{a}$ & $0,0 \mathrm{a}$ \\
\hline T1,5mg & $0,0 \mathrm{a}$ & $0,0 \mathrm{a}$ \\
\hline T3,0mg & $21,66 \mathrm{~b}$ & $40 \mathrm{~b}$ \\
\hline
\end{tabular}

Para analise os dados foram transformados para $\sqrt{ } \mathrm{x}+0,5$.

Medias seguida de letras iguais não diferem estatisticamente pelo teste tukey a $5 \%$ de probabilidade.

Na tabela 8, observa-se que para as variáveis TVEMP1 e TVEMP0, o tratamento utilizado no G1 não difere estatisticamente no utilizado no G2 e o $\mathrm{G} 3$ e difere estatisticamente dos demais.

Tabela 9: Resumo da analise de variância para as variáveis TMan3 (tempo em que o animal permaneceu em escore três para manipulação), TMan2 (tempo em que o animal permaneceu em escore três para manipulação), TMan2 (tempo em que o animal permaneceu em escore dois para manipulação), TVMan1 (tempo que o animal demorou a voltar ao escore um para manipulação).

\begin{tabular}{|l|c|c|c|c|}
\hline & & \multicolumn{3}{|c|}{ Quadrado Médio } \\
\hline F.V. & G.L. & TMan3 & TMan2 & TVMan 1 \\
\hline Trat. & 2 & $2556,33 *$ & $1844,09 *$ & $19947,41 *$ \\
\hline Res. & 26 & 409,32 & 393,55 & 1142,3 \\
\hline CVE & & $49,93 \%$ & $28,76 \%$ & $19,27 \%$ \\
\hline
\end{tabular}

Para analise os dados foram transformados para $\sqrt{ } \mathrm{x}+0,5$.

*Diferenças significativas ao nível de probabilidade de 0,05 . 
Os tratamentos apresentaram diferenças significativas entre si em relação ao tempo em que os animais permaneceram em escore três e dois e no tempo que os espécimes demoraram a retornar ao escore um para o parâmetro manipulação.

Tabela 10 - Estimativa de medias para as variáveis TMan 3 (tempo em que os animais permaneceram em escore 3 para manipulação). TMan 2 (tempo em que o animal permaneceu em escore dois para manipulação), TVMan1 (tempo que o animal demorou a voltar ao escore um para manipulação).

\begin{tabular}{|l|c|c|c|}
\hline & \multicolumn{3}{|c|}{ Médias } \\
\hline Trat. & TMan3 & TMan2 & TVMan1 \\
\hline T1,0 mg & $4,0 \mathrm{a}$ & $15,5 \mathrm{a}$ & $39 \mathrm{a}$ \\
\hline T1,5mg & $16,5 \mathrm{a} \mathrm{b}$ & $27,0 \mathrm{a} \mathrm{b}$ & $72 \mathrm{~b}$ \\
\hline T3,0mg & $36,66 \mathrm{~b}$ & $43,33 \mathrm{~b}$ & $130 \mathrm{c}$ \\
\hline
\end{tabular}

Para analise os dados foram transformados para $\sqrt{ } x+0,5$.

Medias seguida de letras iguais não diferem estatisticamente pelo teste tukey a $5 \%$ de probabilidade.

Segundo a tabela 10, para as variáveis TMan3 e TMan2 o tratamento utilizado no G1 não difere estatisticamente do utilizado no G2; o tratamento realizado no $G 2$ não difere estatisticamente do utilizado no $G 3$ e o tratamento utilizado no G1 difere estatisticamente do empregado no G3.

Para variável TVMan1, todos os tratamento diferem-se estatisticamente entre si.

A tabela 11 revela que a frequência cardíaca dos animais dos três grupos, não sofreram alterações significativas, após a administração do etomidato. 

farmacológica de cágado-de-barbicha Phrynops geoffroanus SCHWEIGGER, 1812 (Testudines, Chelidae). PUBVET, Londrina, V. 6, N. 12, Ed. 199, Art. 1335, 2012.

Tabela 11 - Estimativa de medias para as variáveis FCG1 (frequência cardíaca do G1), FCG2 (Frequência cardíaca dos animais do G2), FCG3 (Frequência cardíaca dos animais do G3).

\begin{tabular}{|l|c|c|c|}
\hline & \multicolumn{3}{|c|}{ Média } \\
\hline Tempo & FCG1 & FCG2 & FCG3 \\
\hline FC0 & $39 a$ & $36,1 \mathrm{a}$ & $35,2 \mathrm{a}$ \\
\hline FC10 & $34,8 \mathrm{a}$ & $34,8 \mathrm{a}$ & $33,1 \mathrm{a}$ \\
\hline FC30 & $36,8 \mathrm{a}$ & $34,3 \mathrm{a}$ & $33,0 \mathrm{a}$ \\
\hline FC60 & $35,1 \mathrm{a}$ & $36,3 \mathrm{a}$ & $31,8 \mathrm{a}$ \\
\hline FC120 & $38,5 \mathrm{a}$ & $36,5 \mathrm{a}$ & $32,2 \mathrm{a}$ \\
\hline FC180 & $37,6 \mathrm{a}$ & $36,0 \mathrm{a}$ & $34,7 \mathrm{a}$ \\
\hline
\end{tabular}

Para analise os dados foram transformados para $\sqrt{ } x+0,5$.

Medias seguida de letras iguais não diferem estatisticamente pelo teste tukey a $5 \%$ de probabilidade.

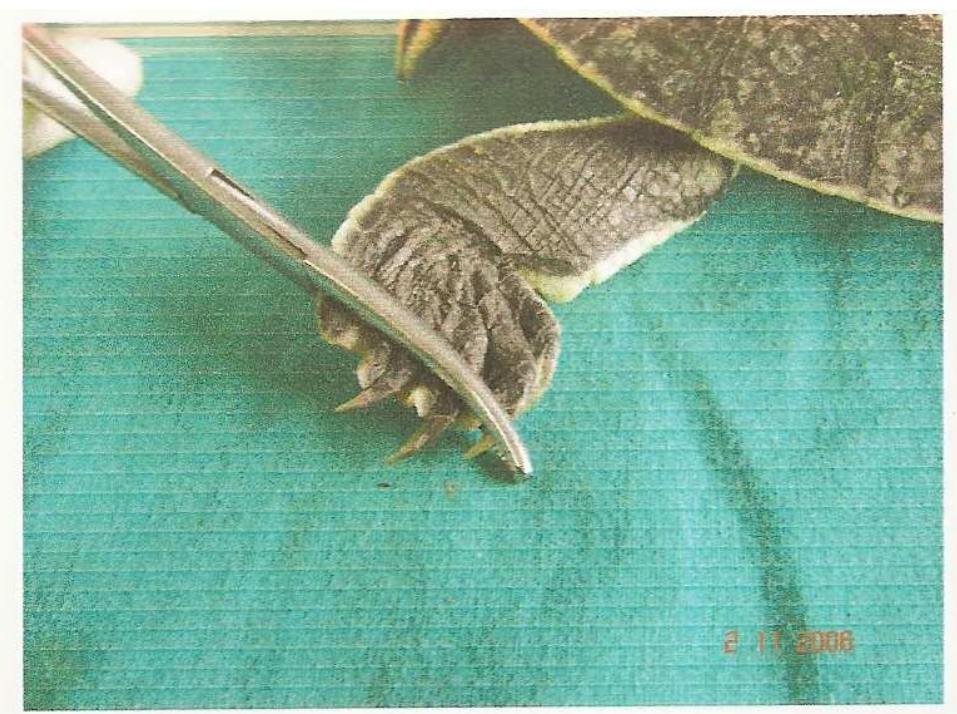

Fotografia 1- Teste de resposta ao estimulo do membro pelvino em um cágado-de-barbicha 
SANTOS, A.L.Q. et al. Avaliação dos efeitos de diferentes doses de etomidatos na contenção farmacológica de cágado-de-barbicha Phrynops geoffroanus SCHWEIGGER, 1812 (Testudines, Chelidae). PUBVET, Londrina, V. 6, N. 12, Ed. 199, Art. 1335, 2012.

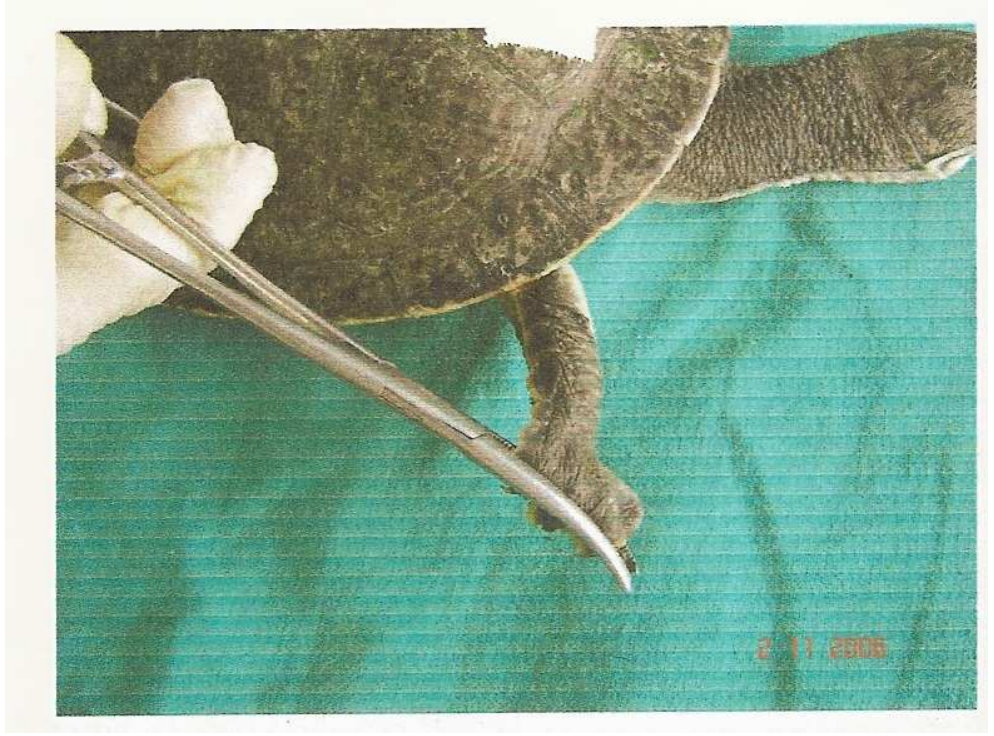

Fotografia 2 - Teste de resposta ao estímulo do membro torácico em Phrynops geoffroanus.

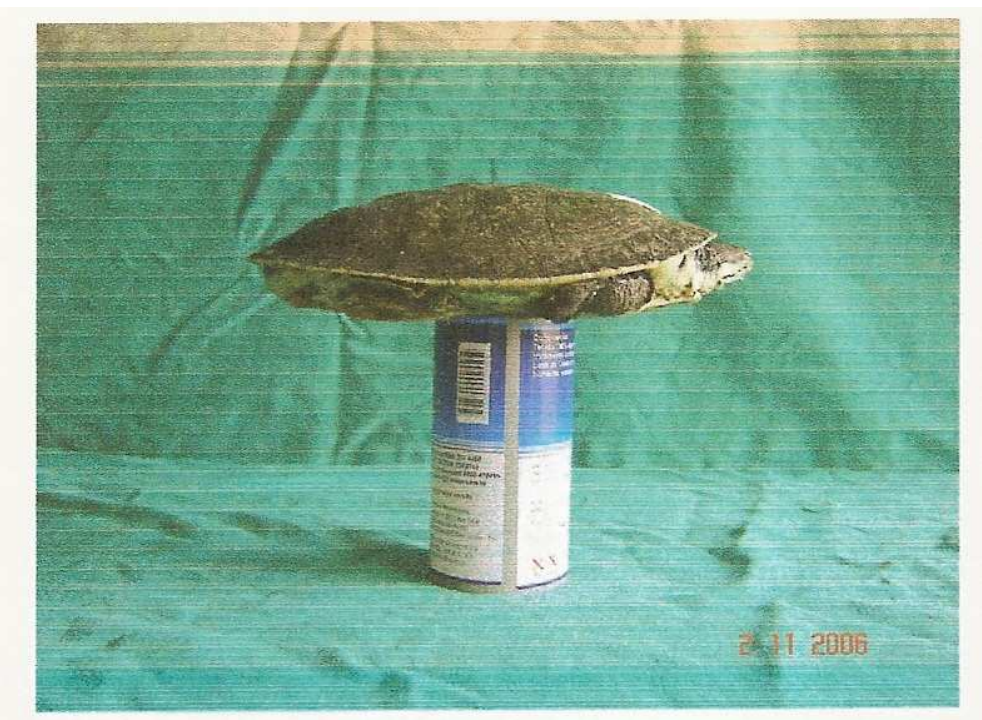

Fotografia 3 - Phrynops geoffroanus com relaxamento muscular em escore um. 
SANTOS, A.L.Q. et al. Avaliação dos efeitos de diferentes doses de etomidatos na contenção farmacológica de cágado-de-barbicha Phrynops geoffroanus SCHWEIGGER, 1812 (Testudines, Chelidae). PUBVET, Londrina, V. 6, N. 12, Ed. 199, Art. 1335, 2012.

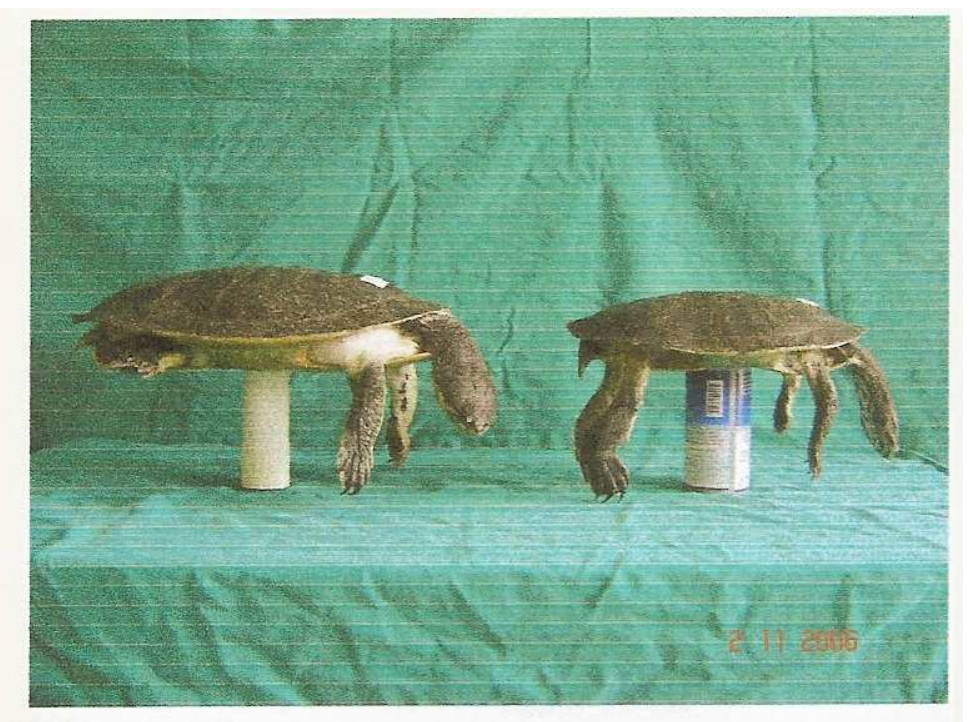

Fotografia 4 - Cágados com relaxamento muscular em escore dois e três, da esquerda para a direita.

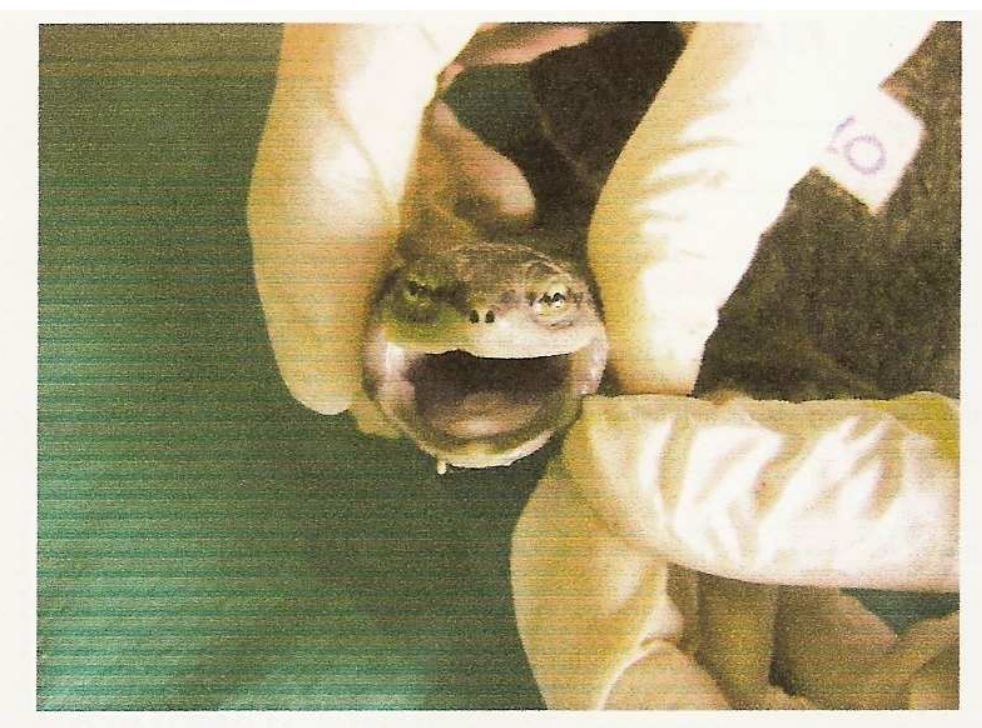

Fotografia 5 - Teste de manipulação em Phrynops geoffroanus. 


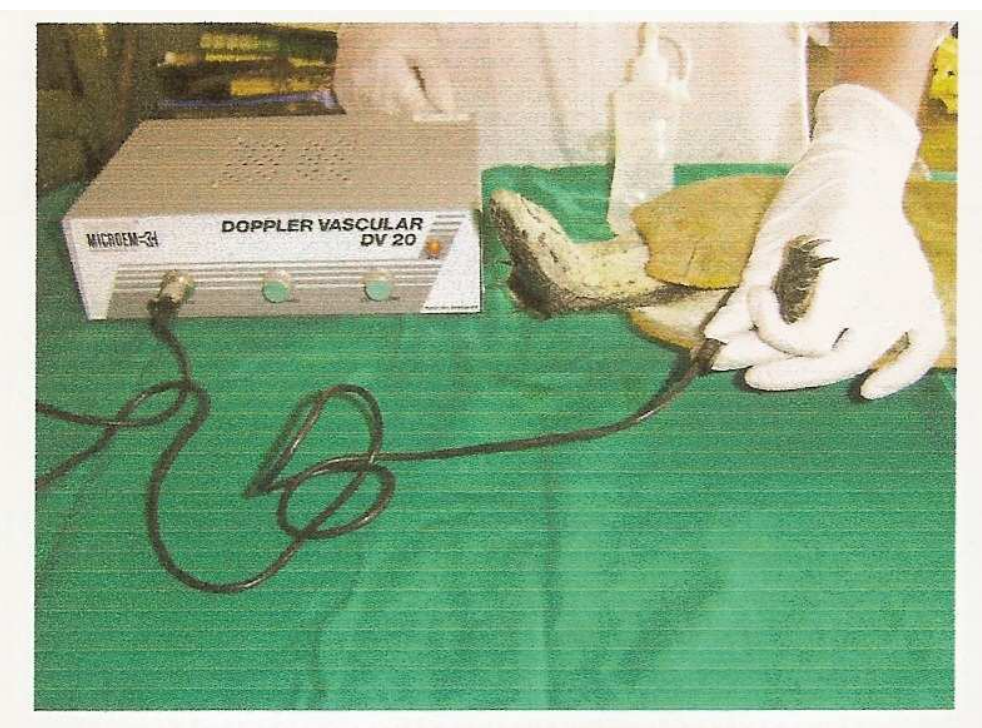

Fotografia 6 - Aferição de batimentos cardíacos com Doppler vascular em cágado-de-barbicha.

\section{DISCUSSÃO}

O estudo foi realizado em ambiente natural dos animais, onde a temperatura oscilou entre 28 e 32 graus Celsius. Essa temperatura é considerada ótima, onde o animal apresenta seu metabolismo em melhor funcionamento (BENNETT, 1991). Portanto, foi esperada a melhor ação possível das drogas utilizadas.

A sedação foi obtida em todos os animais estudados e o período de indução foi menor que dois minutos. Fatos que são justificados pela escolha do anestésico, pois o etomidato é uma droga de rápido efeito (TREVOR; MILLER, 2002), e pela via de administração, pois o seio vertebral cervical pode facilitar a distribuição pelo sistema nervoso. Benson e Forrest (1999) afirmam que substancia de filtragem glomerular não sofrem efeitos farmacocinéticos quando administrados na região pélvica. O metabolismo do etomidato ocorre no fígado e a excreção é por via renal e biliar (EVERS, CROWDER, 2003).

Segundo Leitão et al. (1991), a estabilidade cardiovascular observada após o uso do etomidato, associada ao fato da ausência de liberação de 
histamina, foram as suas principais vantagens inicialmente descritas. Tal afirmação está de acordo com o encontro no experimento, pois em todos os grupos a frequência cardíaca não foi alterada significativamente após a administração do medicamento.

A ausência de analgesia foi detectada nos grupos $G 1$ e G2, pois os animais respondiam ao estimulo dos membros pelvinos quando pinçados. A não resposta ao estimulo dos membros torácicos e pelvinos no G3, em um determinado período do experimento, não é indicativa de ausência de dor e sim do possível bloqueio das vias motoras. Daí sugere-se que o animal sente dor. Porém não consegue retirar o membro.

Bennett (1996) relata que o progresso da anestesia em répteis ocorre em sentido crânio-caudal e durante a recuperação a função motora volta em sentido oposto, quando da administração do anestésico no membro torácico. A ausência da resposta ao estimulo dos membros torácicos, associada à presença de reação ao estimulo dos membros pelvinos nos animais do G1 e G2, pode ser explicada por um maior bloqueio das vias motoras dos membros torácicos devido à proximidade destes do local de administração do anestésico (seio vertebral cervical), em relação aos membros pelvinos.

É prática estabelecida administrar etomidato com fentanila (ALVES; DOREA; ANDRADE, 2002). Entretanto, Bennett (1996) afirma que os opióides são ineficazes nos répteis. Para Buatti e Pasternak (1981), existem diferenças nos receptores opióides entre diferentes espécies. Avila Junior (2005) relata que o butorfanol $(1 \mathrm{mg} / \mathrm{Kg})$ e a fentanila $(0,01 \mathrm{mg} / \mathrm{Kg})$ não acresceram analgesia, na anestesia de Podocnemis expansa, quando associados ao etomidato $(2 \mathrm{mg} / \mathrm{Kg})$. Tais afirmações evidenciam a necessidade de estudos mais profundos a respeito de mecanismos eficientes e bloqueio da dor em répteis.

O G3 foi o único grupo em que todos os animais atingiram escore 11 , ou seja, foram considerados anestesiados. No entanto, o experimento revela que este protocolo não é indicado para anestesia cirúrgica, visto que o tempo 
de permanência dos animais com ausência de resposta ao estimulo dos membros pelvinos (em media 22 minutos), não é considerado satisfatório.

O G1 e G2 não apresentaram diferenças em relação ao tempo em que os animais permaneceram em escore três para os parâmetros locomoção, relaxamento muscular e manipulação. Também foram semelhantes estatisticamente, levando-se em conta o tempo de permanecia em escore um para resposta ao estimulo dos membros torácicos e a constância de resposta ao estimulo dos membros pelvinos, durante todo o experimento. Os resultados obtidos demonstraram que os protocolos utilizados em ambos os grupos são contra-indicados para anestesia cirúrgica, já que todos os cágados não atingiram escore 11, portanto não foram anestesiados, Avila Junior (2005) relata que do grupo com dez animais testados com etomidato $2,0 \mathrm{mg} / \mathrm{Kg}$ associados a butorfanol $1,0 \mathrm{mg} / \mathrm{Kg}$, apenas dois foram anestesiados.

A utilização das doses testadas no $\mathrm{G} 1$ e $\mathrm{G} 2$ é recomendada para procedimentos clínicos e coleta de amostras biológicas em Phrynops geoffroanus, visto que os animais apresentaram perda da capacidade de locomoção, relaxamento muscular satisfatório, bem como facilidade de manipulação. Como não houve diferenças significativas entre os dois grupos pode-se optar pela economia de anestésico utilizando o protocolo do G1 (1,0 $\mathrm{mg} / \mathrm{Kg})$.

Verificou-se que o G1, o G2 e o G3 apresentaram em média 93, 132 e 196 minutos respectivamente de tempo de recuperação. De toda forma, todos os tratamentos podem ter seu tempo de retorno considerado rápido, quando comparados aos anestésicos dissociativos, que apresentaram períodos de recuperação bastante prolongados (SCHILLIGER, 2000), podendo exceder 24 horas.

No G3, um animal recuperou-se da anestesia depois de 24 horas após administração do etomidato, tempo muito superior aos outros animais do mesmo grupo. Fatores individuais como alguma deficiência de metabolização ou excreção da droga, podem justificar o ocorrido (AVILA JUNIOR, 2005). 
A não ocorrência de óbitos demonstrou a segurança dos protocolos e da via de administração adotados. O mesmo resultado foi encontrado por Avila Junior (2005), ao anestesiar Podocnemis expansa com etomidato.

\section{CONCLUSÕES}

Conclui-se que o etomidato, na dose de $3,0 \mathrm{mg} / \mathrm{Kg}$ IV, embora seguro não foi eficiente para anestesia cirúrgica de Phrynops geoffroanus, sendo indicado para ocasiões que necessitem de uma satisfatória contenção farmacológica, em temperatura ótima.

O etomidato, nas doses de $1,0 \mathrm{mg} / \mathrm{Kg}$ IV e $1,5 \mathrm{mg} / \mathrm{Kg}$ IV é indicado em situações que exijam sedação satisfatória em Phrynops geoffroanus, como procedimentos clínicos e coleta de amostras biológicas, em temperatura ótima.

\section{REFERÊNCIAS}

ALVES, T.C.A.; DOREA, E.M.L.; ANDRADE, R.F. Anestésicos Gerais intravenosos. In: SILVA, p. Farmacologia. 6ed. Rio de Janeiro: Editora Guanabara Koogan S.A., 2002, p. 391-403.

AVILA JUNIOR, R.H Efeitos anestésicos do propofol e do etomidato isolados e associados com butorfanol e fentanila em tartaruga-da-amazonia (Podocnimis expansa-Schweigger, 1812). 2005. 31 f. Dissertação (Mestrado)-Faculdade de medicina Veterinária, Universidade Federal de Uberlândia.

BENNETT, R.A. A review of anesthesia and chemical restrain in reptiles. Journal of zoo and Widlife Medicine, Philadelphia, v.22, p.282-303, 1991.

BENNETT, R.A. Anesthesia. In: MADER, D.R. Reptile medicine and sugery. Phyladelphia: W.B. Saunders, 1996, p. 241-247.

BENSON, K.G.; FORREST, L. Characterization of the renal portal system of the commom green iguana (iguana iguana) by digital subtraction imaging. Journal of Zoo and Wildlife Medicine. Philadelphia, v.30, n. 2, p.235-241, 1999.

BOYER, T. H. Clinical anesthesia for reptiles. Bullectin Association of Reptiles Amphibians Veterinarians, Paris, v.2, p. 10-13, 1992.

BUATTI; M.C.; PASTERNAK, G.W. Multiple opiate receptors: phylogenetic differences. Brain Research. V.218 n.1-2, p.400-405. 1981.

ERNEST, C.H.; BARBOUR, R.W. Turtles of the world. Washington: Smithsonian Institution, 1989. 383 p. 
EVERS, A.S.; CROWDER, M. Anéstesicos Gerais. In: HARDMAN, J.G.; LIMBIRD, L.E. Goodman \& Gilman As bases farmacologicas da terapeutica. 10 ed. Rio de Janeiro: McGraw-Hill Internamericana do Brasil LTDA., 2003 p. 257-278.

GARCIA-NAVARRO, C.E.K; PACHALY, J.R. 1994. Manual de hematologia veterinária, São Paulo: Livraria Varela, 1994. 169 p.

HINSCH, H.; GANDAL, C.P. The effects of ethorphine (M-99), oxymorphone hydrochloride and meperidine hydrochloride and meperidine hydrochloride in reptiles. Copeia, p.404-405, 1969.

KANUI, T.I.; HOLE, K. Morphine and pethidine antinociception in the crocodile. Journal of Veterinary Pharmarcological Therapy, v. 15, p. 101-103, 1992.

LEITÃO, F. B. P.; CAMARGO, E. A.; NETO, P. P. R. Anestésicos gerais. In: VALLe, L. B. S.; OLIVEIRA FILHO, R. M.; LUCIA, R.; OLGA, S. Farmacologia Integrada. Rio de Janeiro: Atheneu, 1991, v. 2, p.184-185.

POUGH, F. H.; HEISER, J. B.; MCFARLAND, W. N. A vida dos vertebrados. 2. ed. São Paulo: Atheneu, 1999. 356p.

SCHILLIGER, L. Anesthésie des reptiles - Anesthésie - Reanimation. In: Encyclpédie Vétérinaire. Paris: Editions Scientifiques et Médicales Elsevier. 6p. 2000.

TRAVOR, A.J.; MILLER, R.D. Anestésicos gerais. In: KATZUNG, B.G. Farmacologia Básica e Clínica. 8ed. Rio de Janeiro: Editora Guanabara Koohan S., 2002. P. 367-380. 